\title{
The stellar populations in the central parsecs of galactic bulges
}

\author{
Marc Sarzi ${ }^{1}$, H.-W. Rix, J. C. Shields, L. C. Ho, A. J. Barth, \\ G. Rudnick, A. V. Filippenko and W. L. W. Sargent \\ ${ }^{1}$ University of Oxford, Astrophysics, Keble Road, OX13RH Oxford, UK \\ email: sarzi@astro.ox.ac.uk
}

\begin{abstract}
We present the analysis of Hubble Space Telescope blue spectra at intermediate spectral resolution for the nuclei of 23 nearby disk galaxies. These objects were selected to have nebular emission in their nuclei, and span a range of emission-line classifications as well as Hubble types. Here we focus on the stellar population as revealed by the continuum spectral energy distribution measured within the central $0 .{ }^{\prime \prime} 13(\sim 8 \mathrm{pc})$ of these galaxies. The data were modeled with linear combinations of single-age stellar population synthesis models. The large majority $(\sim 80 \%)$ of the surveyed nuclei have spectra whose features are consistent with a predominantly old $\left(\gtrsim 5 \times 10^{9} \mathrm{yr}\right)$ stellar population. Approximately $25 \%$ of these nuclei show evidence of a component with age younger than $1 \mathrm{Gyr}$, with the incidence of these stars related to the nebular classification. Successful model fits imply an average reddening corresponding to $A_{V} \sim 0.4 \mathrm{mag}$ and stellar metallicity of $(1-2.5) Z_{\odot}$. Our findings reinforce the picture wherein Seyfert nuclei and the majority of low-ionization nuclear emission-line regions (LINERs) are predominantly accretion-powered, and suggest that much of the central star formation in HII nuclei is actually circumnuclear.
\end{abstract}

\section{Introduction}

The centers of galaxies hold special interest as the locations of unusual energetic phenomena. It is now commonly accepted that most galaxies harbor central supermassive black holes (SMBHs) that in the past may have shone as powerful active galactic nuclei (AGNs). The fueling of luminous AGNs requires substantial gas concentrations that might also be favorable sites for star formation, and the formation of a dense star cluster is in fact one means of spawning a SMBH (e.g., Rees 1984). In the present Universe, many galactic nuclei exhibit emission-line activity at low levels that apparently derives from a variable mix of accretion power and star formation (e.g., Ho, Filippenko, \& Sargent 1997). The direct detection of young stellar populations in galactic nuclei is an important means of quantifying star formation in these environments and possible connections to emission-line activity. Nearby examples suggest that recent star formation is not unusual. The Milky Way, IC 342, and NGC 4449 all have central young stellar clusters (Krabbe et al. 1995; Böker et al. 2001, Böker, van der Marel, \& Vacca 1999), and our neighbors M33 and M31 also have blue nuclei that are most likely consistent with the presence of young stars (Lauer et al. 1998). Most existing studies of stellar populations and nebular emission in galaxy centers have been carried out from the ground, and in probing "nuclei" are thus limited to studying regions that may actually be several hundreds of parsecs in scale. Considerable structure may exist on smaller scales, and the spatial distribution of star formation within such a region is of particular interest. A number of ultraviolet (UV) HST surveys have shown that star formation in galactic nuclei often takes place in circumnuclear rings (e.g., Maoz et al. 1996). Furthermore if all bulges harbor a SMBH, there are reasons to believe that it may be difficult to form stars in their very central 
regions. In particular, the tidal field induced by a SMBH may suffice to disrupt molecular clouds before they can gravitationally collapse.

Probing galaxy centers with the highest possible spatial resolution is thus of great value for understanding what powers "nuclear" activity, and how star formation proceeds in these zones. For this reason we undertook a survey of nearby nuclei with the Space Telescope Imaging Spectrograph (STIS) on board HST (the SUNNS survey), acquiring moderate-resolution blue and red spectra for 23 galaxies to study both their nuclear stellar populations and emission-line kinematics, and to detect in particular the presence of very young stars or SMBHs. Quite interestingly, the work of Gonzales Delgado et al. (2004, but see also the contributions by Cid Fernandez \& Rosa Gonzalez Delgado) reaches broadly similar conclusions to what presented here.

\section{The Data}

The nuclear spectral energy distributions (SEDs) modelled here have been extracted from the two-dimensional (2-D) HST-STIS spectra acquired in the course of the SUNNS spectroscopic survey. The SUNNS targets were drawn from the Palomar spectroscopic survey of nearby galaxies (Ho et al. 1997) and include all galaxies within $17 \mathrm{Mpc}$ known to have optical $\mathrm{H} \alpha$ or [NII] $\lambda 6583$ line emission $\left(\gtrsim 10^{15} \operatorname{ergs~s}^{-1} \mathrm{~cm}^{-2}\right)$ within a $2^{\prime \prime} \times 4^{\prime \prime}$ aperture. The sample includes Seyfert galaxies, LINERs, LINER/HiI transition objects, and HiI nuclei. G430L spectra were acquired with the $0 .{ }^{\prime \prime} 2 \times 50^{\prime \prime}$ slit, and 5-pixel-wide $\left(\sim 0 .{ }^{\prime} 25\right)$ extractions were used to generate one-dimensional (1-D) spectra of the nuclei. The resulting aperture of $0 !^{\prime} 2 \times 0 !^{\prime \prime} 25$ is equivalent in area to a circular aperture with radius $r=0 .{ }^{\prime \prime} 13$, corresponding to $8.2 \mathrm{pc}$ for the mean sample distance of $\sim 13 \mathrm{Mpc}$.

\section{The Analysis Method}

To interpret our nuclear spectra, we fit them with synthetic population models that are based on a variety of star formation histories, metallicities, and reddenings. To simulate extended star formation histories, we used linear combinations of single-age stellar population synthesis templates to fit the nuclear spectra directly in pixel space, following Rix et al. (1995). This algorithm derives simultaneously the optimal combination of template spectra and the kinematical broadening required to match the data. By working in pixel space rather than Fourier space, it also allows to exclude wavelength intervals that are contaminated by emission lines. Our library of template spectra is based on the Bruzual \& Charlot (2003, hereafter B\&C) model SEDs for single-age stellar populations with a Salpeter (1955) initial mass function, with 11 different ages spanning 1 Myr to 10 Gyr. In our analysis we use exclusively B\&C model SEDs with solar and 2.5 times solar metallicity. We also included the possibility of interstellar reddening intrinsic to the source. The relative contributions of each single-age template are used to derive luminosity-weighted mean ages $\bar{T}$ and fractions of stars younger than 1 Gyr.

\section{Results}

The primary result of our analysis is that the large majority of the inner $\sim 10 \mathrm{pc}$ of our sample galaxies have spectral energy distributions consistent with simply old stellar populations. Only one object out of 23 is dominated by a young cluster of stars less than 1 Gyr old (Fig. 1, left). When using multi-age population fits to reflect more complex formation histories, we find a distribution of luminosity-weighted mean ages across the sample that is strongly peaked around 10 Gyr. This result persists if we account for 

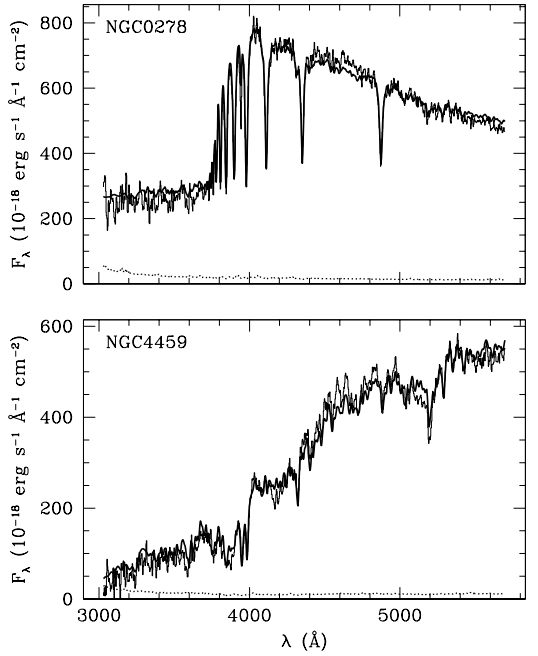

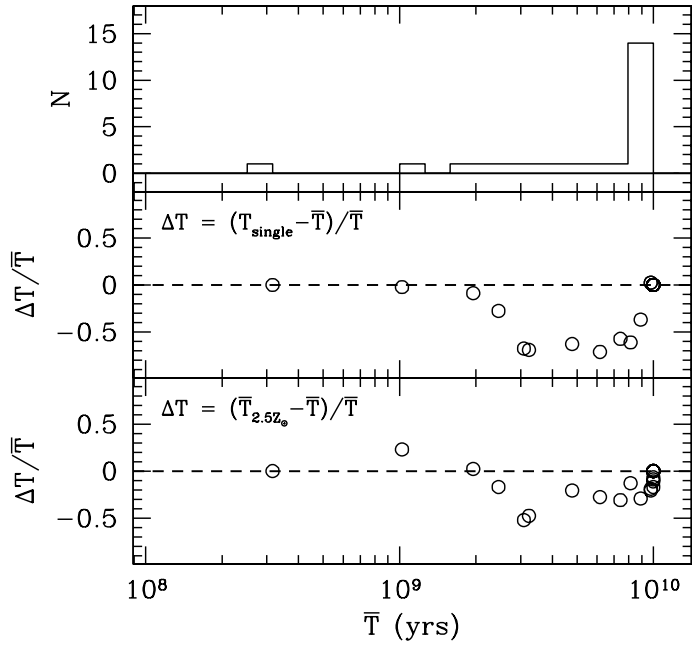

Figure 1. Left: Two examples of nuclear G430L spectra extracted from the central $0 .{ }^{\prime \prime} 25 \times 0 .{ }^{\prime \prime} 2$ of our sample galaxies. The dotted lines at the bottom of each panel show the error array corresponding to the observed fluxes, while the solid thick lines show our best-fitting multiple-starburst population models with solar metallicity. The observed fluxes have been corrected for Galactic foreground extinction, while the models account for dust extinction intrinsic to the source. NGC278 is the only galaxy in our sample which nucleus is clearly dominated by young ( $300 \mathrm{Myr})$ stars, while NGC4459 is more representative for the typically old nuclear stellar population of bulges. The poor fit to the CN-band absorbtion feature around $4200 \AA$ highlights the need for models with non-solar abundance ratios. Right: Distribution of the luminosity-weighted age estimates obtained from the best-fitting multiple-starburst models (top panel), which are also compared to the age of the best fitting single-age templates (middle panel) and the luminosity-weighted ages from multiple-starburst models of super-solar metallicity (bottom panel). Even considering quite extreme metallicities $\left(Z=2.5 Z_{\odot}\right)$ the $\bar{T}$ values in average shift by less than $30 \%$ towards younger ages.

dust reddening and super-solar metallicities as contributors to, or possible alternative explanations for, the quite red observed spectra (Fig. 1, right). The models also imply average reddening values of $A_{V} \sim 0.4 \mathrm{mag}$, stellar metallicity of $(1-2.5) Z_{\odot}$, and suggest an $\alpha$-enhancement in the chemical content of many of these nuclei, which would result from a rapid star formation.

There is, however, a significant fraction of galactic nuclei that show modest amounts of young stars: evidence for stars younger than 1 Gyr is found in $\sim 25 \%$ of the cases. Very young stars, $\sim 1 \mathrm{Myr}$, are not required by the spectral template fits for any object, and their possible contribution to the flux at the blue end of our spectra $(\sim 3100 \AA)$ is limited to less than $6 \%$ on average. With these upper limits, the production of ionizing photons by very young stars falls short of the requirements for powering the nebular emission in the central $10 \mathrm{pc}$ of the Seyfert nuclei and most of the LINERs in our sample (Fig. 2, left). Furthermore, none of these 23 galactic nuclei contain enough very young stars to explain the nebular luminosity observed over $\sim 100$ pc scales, as typically observed from the ground. This shortfall of stellar UV photons holds true regardless of internal dust extinction. The presence of a young population ( $\lesssim 1$ Gyr) appears, however, correlated with the classification of the emission-line ratios (Fig. 2, right).

In Seyferts and most LINERs our findings are naturally explained, if ionization comes largely from AGN accretion, not stars. The association of young population with transition objects suggest that stellar phenomena may have some role in influencing the 

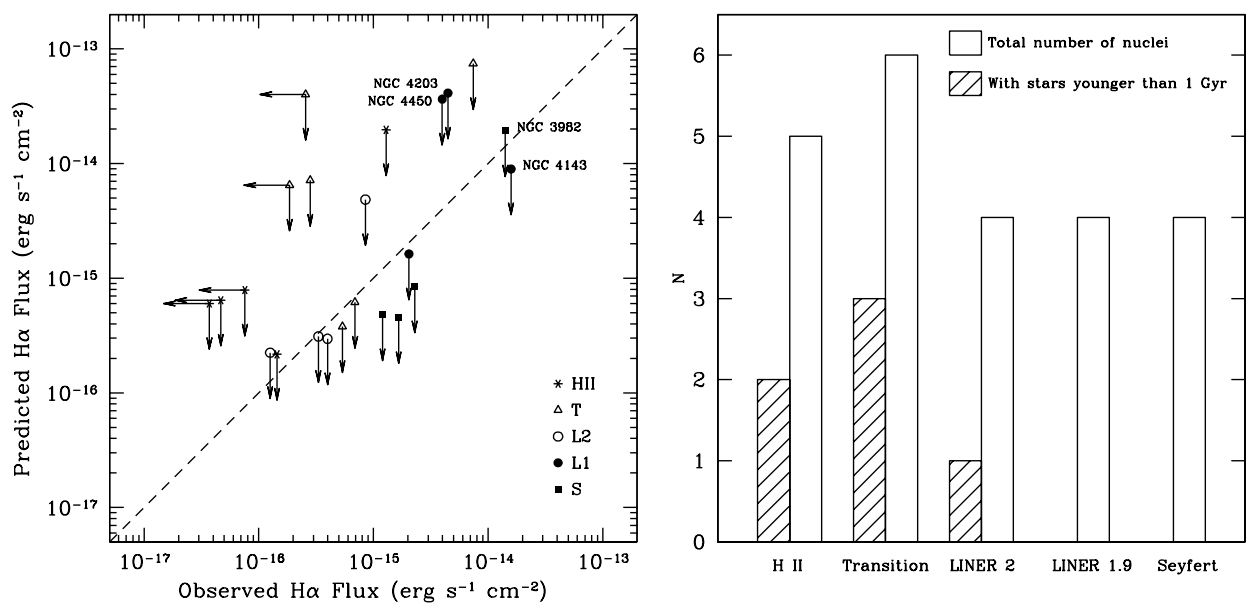

Figure 2. Left: Comparison of the $\mathrm{H} \alpha$ fluxes predicted for HII regions powered by the maximum number of young stars indicated by our G430L spectra, and the observed $\mathrm{H} \alpha$ emission in our G750M spectra. The symbol type denotes the ground-based, nuclear emission-line classification of our objects, while the dashed line shows the unity, consistency limit. Sources in which the stellar fluxes are probably overestimated due to contamination by an AGN continuum are labeled (NGC 3982, NGC 4143, NGC 4203, and NGC 4450). Correction of the observed H $\alpha$ emission for internal extinction inferred from the observed decrement of the Balmer emission lines will further shift most plotted points to the right. Considering that emission-line fluxes obtained in ground-based measurements (Ho et al. 1997) contain 10-100 times as much emission as measured here, none of these galactic nuclei contain enough very young stars in their central $\sim 10$ pc to explain the nebular luminosity observed over $\sim 100$ pc scales. Right: Incidence of stars younger that $1 \mathrm{Gyr}$ in the surveyed galactic nuclei as a function of their ground-based spectral classification.

observed emission, although the comparison between predicted and observed $\mathrm{H} \alpha$ fluxes implies that other energetic processes than photoionization by O-type stars would have to be involved. For HiI nuclei, our results imply that much of the central star formation is actually circumnuclear - it occurs in the range of a few $10 \mathrm{pc}$ to a few $100 \mathrm{pc}$.

We note in closing that even for nearby galaxies at $H S T$ resolution, the contamination from stars of the central portion of the surrounding bulge is an important limiting factor in determining the stellar content of galactic nuclei. We could not have detected a quantity of young stars as small as found at the center of the Milky Way.

\section{References}

Böker, T., van der Marel, R. P., \& Vacca, W. D. 1999, AJ, 118, 831

Böker, T., van der Marel, R. P., Mazzuca, L., Rix, H.-W., Rudnick, G., Ho, L. C., \& Shields, J. C. 2001, AJ, 121, 1473

Bruzual, G. \& Charlot, S. 2003, MNRAS, 344, 1000

González Delgado, R. M., et al. 2004, ApJ, 605, 127

Ho, L. C., Filippenko, A. V., \& Sargent, W. L. W. 1997, ApJS, 112, 315

Krabbe, A., et al. 1995, ApJ, 447, L95

Lauer, T. R., Faber, S. M., Ajhar, E. A., Grillmair, C. J., \& Scowen, P. A. 1998, AJ, 116, 2263

Maoz, D., Barth, A. J., Sternberg, A., Filippenko, A. V., Ho, L. C., Macchetto, F. D., Rix, H.-W., \& Schneider, D. P. 1996, AJ, 111, 2248

Rees, M. J. 1984, ARA\&A, 22, 471

Rix, H.-W., Kennicutt, R. C., Braun, R., \& Walterbos, R. A. M. 1995, ApJ, 438, 155

Salpeter, E. E. 1955, ApJ, 121, 161 